\title{
Verein der Augenärzte von Ost- und Westpreußen
}

Tagung am 30. XI. 1929 in der Univ.-Augenklinik Königsberg i. Pr.

C. H. Sattler (Königsberg i. Pr.): Glaukom, Basedowsche Krankheit und Sympathikus.

Bei einer 7ojährigen Dame entwickelte sich auf der Grundlage einer seit zehn Jahren bestehenden rechtsseitigen Struma eine Basedowsche Krankheit mit nur rechtsseitigem Exophthalmus und rechtsseitigem Glaukom. Das rechte Auge mit einem Druck von $47 \mathrm{~mm} \mathrm{Hg}$ zeigte eine außerordentlich starke Erweiterung der episkleralen Gefäße. Der Augendruck sank rechts während der einige Sekunden dauernden Druck-messung um 8-16 mm Hg, während das linke Auge bei Messung mit dem gleichen Gewicht in der gleichen Zeit nur $2 \mathrm{~mm}$ weicher wurde. Nach drei Röntgenbestrahlungen (zwei Felder Schilddrüse, ein Feld Thymus zu je 75\% HED) verschwand rechts innerhalb einiger Tage das Glaukom, das durch Miotika kaum zu beeinflussen gewesen war, und wurde das Auge blaß. Der Exophthalmus bildete sich rasch zurück.

Sattler weist zur Erklärung des Zusammentreffens von rechts-seitiger Struma, Exophthalmus und Glaukom auf die Beziehungen dieser drei Krankheitserscheinungen zum Halsstrang des Sympathikus hin. Die ungewöhnlich starke Erweiterung der episkleralen Gefäße, die Stei-gerung des Augendrucks und dessen rasche Abnahme durch das Gewicht des Tonometers scheinen ihm im Zusammenhang mit einer vom Sympathikus ausgelösten Erweiterung der Augengefäße zu stehen. Er glaubt, daß die günstige Wirkung der Röntgenbestrahlung nicht durch eine Beeinflussung der innersekretorischen Funktion von Schilddrüse und Thymus, sondern durch eine Einwirkung auf den Halsstrang des Sympathikus bedingt war, denn trotz des raschen Rückgangs von Glaukom und Exophthalmus zeigte die Grundumsatzsteigerung keine wesentliche Anderung.

Gredsted: Über einäugiges Doppeltsehen.

Nach einer kurzen Übersicht über verschiedene physikalische Ur-sachen für einäugiges Doppeltsehen folgt der ausführliche Bericht über einen Fall von Strabismus convergens, bei dem nach der Operation Doppelbilder auf dem vorher schielenden Auge auftraten. Das Trugbild erschien gekreuzt in einem Abstande von $30^{\circ}$ entsprechend dem früheren Schielwinkel. Während der 1 1 12 jährigen Beobachtungszeit wurde eine allmähliche Annäherung der beiden Bilder des Schielauges festgestellt.

Das monokulare Doppeltsehen ist in diesem Fall durch die Aus-bildung einer zweiten Fovea, einer sogenannten Pseudofovea, zu erklären. Wqährend der Zeit des Schielens hatte sich eine neue Sehrichtungsgemein-schaft beider Augen, eine sogenannte falsche Korrespondenz entwickelt, die nach Korrektion der Schielstellung zur Wahrnehmung paradoxer Doppelbilder führte.

Kurzer Überblick über die Literatur. 244 Verein der Augenärzte von Ost- und Westpreußen. Jahns: Die Größe des Schielwinkels. (Erscheint später ausführlich.) Kunz: Die Granulose-Frequenz in Ostpreußen in den letzten Jahren naeh dem Material der Kónigsberger Univ.-Augenklinik,, (Siehe diese Zeitschrift Bd. 71, S. 217.) 
Bírch-Hirschfeld bespricht im Anschluß an den Bericht von Kunz den Stand der

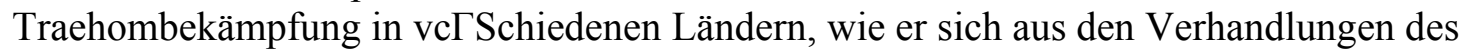
Internat. Ophthalmologenkongresses in Amsterdam ergab. (Siehe diese Zeitschrift Bd. 71, S. 213.)

Feeder- Demonstration eines 17 Jahre alten Pat. mit typischem Biedîsc $7 / 8$ ten Syndrom: Dystrophia adiposo-genitalis, Polydaktylie (sechs Finger und sechs Zehen beiderseits), Retinitis pigmentosa mit Optikus-atrophie und geistiger Entwicklungshemmung. Das Röntgenbild der Sella turcica ergab keine Besonderheiten. Anamnestisch väterlicherseits seit Generationen Trunksucht. Vater Potator. Mutter bereits im 35. Lebensjahr in die Menopause gekommen, leidet seitdem an patho-logischer Fettsucht. Ein i4Jähriger Bruder leidet gleichfalls an einem voll ausgebildeten Biedlschen Syndrom. Zwei weitere Geschwister sind gesund. Eine früh verstorbene Schwester soil sechs Finger gehabt haben.

Vereinigte Medizinische Gesellschaît in Kiew, Sektion für Augenheilkunde.

Sitzung vom 11. Januar 1930. Vorsitzender: Prof. M. Lewitsky. 1. M. Lewitsky: Eigentümliche Resultate naeh der Röntgentherapie des Ulcns rodens.

Bei einem Kranken von 30 Jahren mit Ulcus rodens des medialen Lidwinkels heilte das Geschwür naeh der Röntgentherapie oberflächlich vollständig, während in der Tiefe, unter der Haut, der Prozeß fort-gesetzt progressierte.

W. Kramarewsky: Fall von Membrana pupillaris persistens corneae adhaerens oc. dext. R. A.: Conjunctivitis cat. Hornhauttrübung. Im Zentrum der Hornhaut ist die Trübung größer und wird zirkulär von Pigmentpünktchen umgeben. Von der Irisoberfläche ziehen feine Fäden zu diesen Pigmentpünktchen. SpaUlampenbefund: Hornhaut rechts doppelt so dick wie, links. Von der vorderen Irisoberfläche und vom Pupillarrande ziehen feine braune Fäden naeh der Hornhaut hin, wo sie sich an ihrer Hinterfläche (an den Pigmentpunkten) befestigen. Im anderen sind Hornhaut, Pupille und Linse o. B. Keine hinteren Synechien.

Auf dem linken Auge einfache Membrana pupillaris perseverans. Aussprache: Korenjewitsch, Samkowsky, Danilewsky.

S. Sitzung vom 20. Januar 1930. - Vorsitzender: Prof. M. Lewitsky. 1. Tichonowitsch demonstriert einen Fall bei dem durch das operative Iriskolobom ein stark ausgeprägter Processus ciliares sehr gut zu sehen ist. 